\title{
Kinetic Sensitivity of a New Lumbo-Pelvic Model to Variation in Segment Parameter Input
}

\author{
Helen Crewe, ${ }^{1}$ Amity Campbell, ${ }^{2}$ Bruce Elliott, ${ }^{1}$ and Jacqueline Alderson ${ }^{1}$ \\ 1 University of Western Australia; ${ }^{2}$ Curtin University
}

\begin{abstract}
This study aimed to assess variability in lumbo-pelvic forces and moments during a dynamic high-impact activity (cricket fast bowling) when calculated using different body segment parameters (BSPs). The first three BSPs were estimated using methods where the trunk was divided into segments according to nonspinal anatomical landmarks. The final approach defined segment boundaries according to vertebral level. Threedimensional motion analysis data from nine male cricketers' bowling trials were processed using the four BSPs. A repeated-measures analysis of variance revealed no significant effect on peak lumbo-pelvic forces. However, the segmentation approach based on vertebral level resulted in significantly larger peak flexion and lateral flexion moments than the other BSP data sets. This has implications for comparisons between studies using different BSPs. Further, given that a method defined with reference to vertebral level more closely corresponds with relevant anatomical structures, this approach may more accurately reflect lumbar moments.
\end{abstract}

Keywords: inertial parameters, lumbar load, motion analysis

\begin{abstract}
Athletes who participate in sports that require repetitive hyperextension of the trunk, particularly in combination with rotation, such as tennis, cricket fast bowling, javelin throwing and gymnastics, have an increased risk of developing a stress fracture of the pars interarticularis in the lumbar spine, known as spondylolysis. ${ }^{1,2}$ Given that fast bowling technique has been linked with the onset of spondylolysis ${ }^{3}$ a significant body of research has been centered around kinematic analysis of the bowling action. However, there exists little investigative interest in the potential relationship between lumbar kinetics during fast bowling and injury incidence, despite the lower lumbar region being the most common site of injury inclusive of spondylolyses. ${ }^{1}$ It is the application of load to the lumbar vertebrae that results in cumulative microtrauma and eventual bony failure, ${ }^{4,5}$ and as such, knowledge of the associated lumbar kinetics during fast bowling is critical for greater understanding of the causal, loading related mechanisms of spondylolysis.

The paucity of kinetic information reporting lumbar forces may be attributed to the difficulty associated with measuring the required biomechanical inputs. These include a suitable motion tracking system to estimate lower limb, pelvis and lumbar spine position
\end{abstract}

Helen Crewe, Bruce Elliott, and Jacqueline Alderson (Corresponding Author) are with the School of Sport Science, Exercise and Health, University of Western Australia, Perth, Australia. Amity Campbell is with the School of Physiotherapy and Curtin Health Innovation Research Institute, Curtin University, Perth, Australia. and orientation, together with the estimation of each segment's inertial characteristics (mass, center of mass and radius of gyration). However, advances in motion analysis technology has resulted in improved accuracy in the recording and calculation of lumbar spine region kinematics ${ }^{6}$ and therefore this issue will not be discussed further in this manuscript. In contrast, the body segment parameter (BSP) input data used in the kinetic calculation remains inconsistent (Table 1). ${ }^{6-9}$

Traditional BSPs have estimated segment characteristics using data derived from cadaveric specimens with the pelvis and trunk combined as a single mass and divided into either two ${ }^{10}$ or three ${ }^{11}$ segments. The rationale for the boundary definitions was based on the location of body organs (with the intention for them to remain intact following dissection) and the proximity of easily identifiable surface landmarks. However, these segment definitions may not be ideal for calculation of lumbar loads for the following reasons. First, with respect to vertebral level, the location of the anatomical landmarks used to define the segment boundaries (typically the umbilicus and xyphoid process) is highly variable between individuals. ${ }^{12}$ Second, the boundary definitions do not allow for clear delineation of the pelvis, lumbar and thoracic regions. Indeed, Zatsiorsky's most suitable lumbar region segment-named the "lower trunk," incorporates the pelvis and the lower part of the lumbar spine, while the "middle trunk" segment incorporates the upper part of the lumbar spine and lower part of the thoracic spine. This later point is particularly pertinent given the stark differences in the thoracic and lumbar vertebral regions both anatomically 
Table 1 Body segment parameter input data applied in previous research to calculate lumbo-pelvic kinetics

\begin{tabular}{lll}
\hline Study & Movement Analyzed & Body Segment Parameters Used \\
\hline Khoo et al $(1995)^{8}$ & Walking & Zatsiorksy et al (1986) \\
Shum et al $(2007)^{7}$ & Sit-to-Stand & Shan et al (2003) \\
Seay et al $(2008)^{6}$ & Running & Pearsall et al (1996) \\
Ferdinands et al $(2009)^{9}$ & Cricket Fast Bowling & De Leva (1996) \\
\hline
\end{tabular}

and functionally. ${ }^{13}$ It is therefore important to differentiate between the lumbar and thoracic spinal regions in motion analysis. This may be possible using BSPs provided by Pearsall et al, ${ }^{14}$ who used computed tomography scanning of living subjects and defined BSPs using segment boundaries with reference to vertebral level.

The magnitude of the effect of employing different BSPs on lumbo-pelvic kinetic calculations is unknown. Therefore, the aim of this study is to analyze the sensitivity of the calculation of lumbo-pelvic forces and moments during fast bowling, to four sets of BSPs. This includes three commonly used traditional methods, ${ }^{15-17}$ and the more recent method of Pearsall and colleagues (1996) that utilizes vertebral level segment boundaries. ${ }^{14}$

\section{Methods}

\section{Subjects}

Nine asymptomatic right-arm fast bowlers from district and/or state junior cricket squads volunteered to participate in the study. All bowlers were male Caucasians (mean age 16.9 years, height $181 \mathrm{~cm}$, weight $69.4 \mathrm{~kg}$ ). Ethical approval was obtained from the University of Western Australia's Human Research Ethics Committee and all participants (and their guardians, where required) provided informed, written consent to participate in this research.

\section{Testing Procedure}

Data collection was performed at the biomechanics laboratory at the School of Sport Science, Exercise and Health at the University of Western Australia. A 12-camera Vicon MX motion analysis system (Vicon Peak, Oxford, UK) operating at $250 \mathrm{~Hz}$ and a $1.2 \mathrm{~m} \times$ $1.2 \mathrm{~m}$ force plate (Advanced Mechanical Technology Inc., Watertown, MA) sampling at $2000 \mathrm{~Hz}$ were used to collect kinematic and ground reaction force (GRF) data. A cricket crease was located to ensure the bowler's front foot (the support limb during the period of greatest impact) landed on the force plate. After carrying out a self-directed warm-up, participants were required to bowl 3 overs (18 deliveries) at match pace. Three successful trials comprising the highest ball release speed were selected for analysis.

\section{Data Collection and Processing}

Retro-reflective markers were affixed to the participants' skin and shoes according to a customized marker set and model for the lower limbs and pelvis. ${ }^{18}$ This comprised of single markers placed on the head of the first and fifth metatarsals, calcaneus, anterior superior iliac spines and posterior superior iliac spines. A series of triad marker clusters, consisting of three markers attached to a semirigid plastic baseplate, were attached bilaterally to the thigh and lower legs. Subject-specific static calibration trials were performed using a foot-calibration rig to measure foot abduction/adduction and inversion/eversion angles. ${ }^{18}$ Additionally, static trials were collected with markers placed on the medial and lateral malleoli and medial and lateral femoral condyles, with dynamic functional methods adopted to determine the knee joint axes and hip joint centers. ${ }^{18}$ This approach enabled the pelvis and all lower limb joint centers and axes of rotation to be determined and relevant anatomical coordinate systems to be defined.

To define the lumbar segment, markers were placed on the L1, L3, L5 spinous processes and approximately $5 \mathrm{~cm}$ on either side of the spine at the level of L2 and L4 (LUL, RUL, LLL, RLL) (Figure 1). The L5 marker was used to represent the origin of the lumbar segment coordinate system. The y axis was defined using a vector from the L5 to L1 marker, the $\mathrm{x}$ axis was calculated from the cross product of the $y$ axis, and a defining line between the LLL and RLL markers. Finally, the $\mathrm{z}$ axis was calculated as the cross product of the $y$ and $x$ axes. The lumbo-pelvic joint location was defined using previously established methods ${ }^{6}$ and served as the point of application for the inverse dynamics analysis of the lumbo-pelvic segment.

Data were filtered using a fourth-order low-pass Butterworth filter operating at a cut-off frequency of 15 $\mathrm{Hz}$ for the marker trajectories and $50 \mathrm{~Hz}$ for the GRF data. Cutoff frequencies were determined using residual analysis techniques. ${ }^{19}$

A mathematical model that used inverse dynamics and scaled inertial parameters for the lower limb segments ${ }^{15}$ was extended to incorporate pelvis and lumbar segments for the calculation of lumbo-pelvic kinetics. The trials were processed four separate times using different BSP data for the pelvis and lumbar segments: (1) de Leva (1996), ${ }^{15}$ (2) Shan et al (2003), ${ }^{16}$ (3) Pearsall 


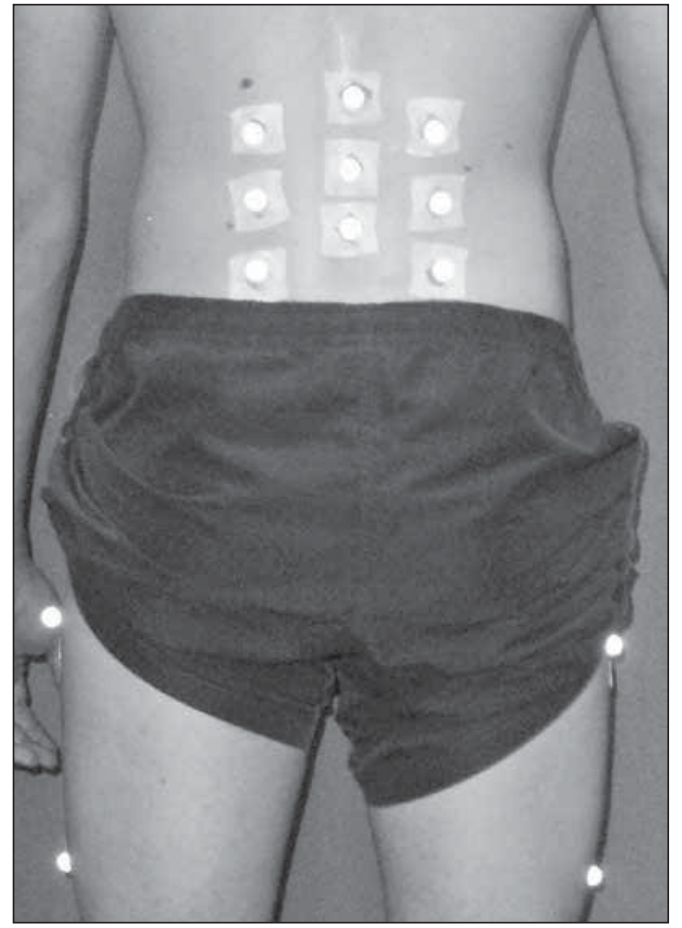

Figure 1 - The retro-reflective marker set on a subject's lumbar spine and posterior pelvis. From top: L1, left and right $5 \mathrm{~cm}$ lateral to L2 spinous process (LUL and RUL); L3, left and right $5 \mathrm{~cm}$ lateral to L4 spinous process (LLL and RLL); $\mathrm{L} 5$, and left and right posterior superior iliac spines.

et al (1994), ${ }^{17}$ and (4) Pearsall et al (1996) ${ }^{14}$ (Tables 2 and 3). All BSPs were based on Caucasian participant data.

\section{Statistical Analysis}

Statistical analysis was performed using SPSS 19.0 (SPSS Inc., Chicago, IL.). Following assessment of the data for normality, homogeneity of variance and sphericity, one-way repeated measures analyses of variance were used to evaluate the effect of BSP on peak lumbar forces and moments. Fisher's least significant difference post hoc tests were used to determine where differences occurred. Statistical significance was set at $P<.05$.

\section{Results}

The repeated measures analysis of variance revealed no differences in discrete peak lumbar forces calculated in the phase between front foot contact and ball release, using the four BSP data sets (Table 1). Further, there was no difference in the wave form of the continuous data during this phase (Figure 2). Conversely, there was a statistically significant overall effect of BSP on the calculated peak flexion moment $(F=20.151, P<.001)$, left lateral flexion moment $(F=30.880, P<.001)$ and right rotation moment $(F=11.477, P<.001)$. Post hoc analyses revealed that each BSP resulted in significantly different peak flexion and lateral flexion moments than the other three BSP's $(P<.05)$, with the moments calculated using the Pearsall et al (1996) BSP inputs being the largest. There was no difference between peak rotation moments when calculated using de Leva (1996), Pearsall et al (1994) or Pearsall et al (1996) BSPs, but when using input data from Shan et al (2003) the peak rotation moment was significantly smaller than the former three $(P<.001)($ Table 4$)$

\section{Discussion}

This study assessed the difference in peak lumbar forces and moments during fast bowling when calculated using different BSP inputs. Of the four BSP data sets compared, three_-de Leva (1996), Shan et al (2003), and Pearsall et al (1994) — were based on trunk segments that did not specifically delineate the pelvis and lumbar regions. One of the difficulties associated with kinetics calculations for the lumbo-pelvic region is the limited lumbar and pelvis inertial parameter data available. To date, only one study ${ }^{14}$ has provided specific lumbar and pelvis BSPs, using computed tomography to estimate the mass, volume, density, center of mass and moment of inertia of the pelvis, lumbar (L1-L5) and thoracic segments (T1-T12). The use of these BSP data (Pearsall et al, 1996) to compute lumbar

Table 2 Inertial parameters for the pelvis / lower trunk segments

\begin{tabular}{|c|c|c|c|c|c|c|c|}
\hline & \multirow{2}{*}{$\begin{array}{l}\text { Segment } \\
\text { Boundaries }\end{array}$} & \multirow{2}{*}{$\begin{array}{l}\text { Measurement } \\
\text { Technique }\end{array}$} & \multirow{2}{*}{$\begin{array}{c}\text { Mass } \\
(\% \mathrm{BM})\end{array}$} & \multirow{2}{*}{$\begin{array}{l}\text { CM } \\
(\%)^{b}\end{array}$} & \multicolumn{3}{|c|}{ Radius of Gyration } \\
\hline & & & & & $\mathbf{x}$ & $y$ & $\mathbf{z}$ \\
\hline De Leva $(1996)^{15}$ & Umbilicus-HSPI ${ }^{\mathrm{a}}$ & Gamma Ray & 11.17 & 61.15 & 61.5 & 58.7 & 55.1 \\
\hline Pearsall et al (1994) $)^{17}$ & Umbilicus-Pubic Symphysis & MRI & 13.3 & 44.7 & 56.9 & 56.9 & 44.5 \\
\hline Pearsall et al $(1996)^{14}$ & S1-S5 & CT & 10.7 & 59.7 & 66.7 & 68.9 & 42.2 \\
\hline Shan et al $(2003)^{16}$ & Umbilicus-HSPI $^{\mathrm{a}}$ & Body Surface Profile Scan & 12.5 & 42.6 & 39.6 & 38.2 & 34.0 \\
\hline
\end{tabular}

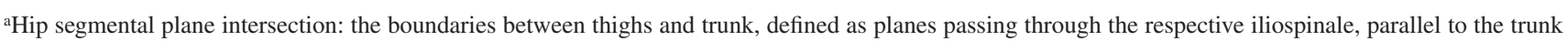
sagittal axis, and forming a $37^{\circ}$ angle with the sagittal plane. ${ }^{15}$

bFrom cranial end of the segment. 
Table 3 Inertial parameters for the abdomen/middle trunk segments

\begin{tabular}{|c|c|c|c|c|c|c|c|}
\hline & \multirow{2}{*}{$\begin{array}{l}\text { Segment } \\
\text { Boundaries }\end{array}$} & \multirow{2}{*}{$\begin{array}{l}\text { Measurement } \\
\text { Technique }\end{array}$} & \multirow{2}{*}{$\begin{array}{c}\text { Mass } \\
\text { (\% BM) }\end{array}$} & \multirow{2}{*}{$\begin{array}{l}\text { CM } \\
(\%)^{a}\end{array}$} & \multicolumn{3}{|c|}{ Radius of Gyration } \\
\hline & & & & & $\mathbf{x}$ & $y$ & $z$ \\
\hline De Leva $(1996)^{15}$ & Xyphoid-Umbilicus & Gamma Ray & 16.33 & 45.02 & 48.2 & 46.8 & 38.3 \\
\hline Pearsall et al $(1994)^{17}$ & Xyphoid-Umbilicus & MRI & 16 & 49 & 66.4 & 66.4 & 50.9 \\
\hline Pearsall et al $(1996)^{14}$ & L1-L5 & $\mathrm{CT}$ & 12.1 & 56.3 & 82.4 & 87.7 & 70.6 \\
\hline Shan et al $(2003)^{16}$ & Xyphoid-Umbilicus & Body Surface Profile Scan & 12.4 & 48.9 & 55.5 & 54.4 & 40.2 \\
\hline
\end{tabular}

aFrom cranial end of the segment.

Table 4 Peak lumbar forces and moments for the four BSP data sets

\begin{tabular}{|c|c|c|c|c|}
\hline & DL & SB & P94 & P96 \\
\hline & Mean \pm SD & Mean \pm SD & Mean \pm SD & Mean \pm SD \\
\hline \multicolumn{5}{|l|}{ Force $(\mathrm{N})$} \\
\hline Anteroposterior & $-2364.6 \pm 850.1$ & $-2313.0 \pm 835.5$ & $-2472.0 \pm 889.7$ & $-2208.4 \pm 799.2$ \\
\hline Vertical & $1964.8 \pm 872.4$ & $1919.8 \pm 857.5$ & $1997.5 \pm 900.1$ & $1889.2 \pm 840.0$ \\
\hline Mediolateral & $2098.8 \pm 384.2$ & $1936.7 \pm 348.6$ & $2127.7 \pm 382.3$ & $1887.1 \pm 346.2$ \\
\hline \multicolumn{5}{|l|}{ Moment $(\mathrm{N} \cdot \mathrm{m})$} \\
\hline Flexion & $923.4 \pm 259.0^{*}$ & $674.1 \pm 179.3 *$ & $1223.2 \pm 428.3^{*}$ & $1460.2 \pm 572.4 *$ \\
\hline Lateral Flexion & $1289.3 \pm 374.5^{*}$ & $926.4 \pm 261.5^{*}$ & $1544.2 \pm 324.0^{*}$ & $1819.1 \pm 416.1 *$ \\
\hline Rotation & $-1555.0 \pm 551.7$ & $-951.1 \pm 264.1 *$ & $-1520.8 \pm 465.6$ & $-1515.0 \pm 413.4$ \\
\hline
\end{tabular}

Note. DL, de Leva (1996); SB, Shan et al (2003); P94, Pearsall et al (1994); P96, Pearsall et al (1996).

*Statistically significant from the other three BSPs, $P<.05$.

kinetics during fast bowling resulted in significantly larger peak flexion and lateral flexion moments when compared with the kinetic outputs determined using the other three traditional BSP data sets. The relevance of these findings is not specific to fast bowling investigations, but has implications for researchers wishing to calculate lumbar kinetics during other activities.

It is accepted that BSP estimates are highly sensitive to the location of the boundaries used to define segments ${ }^{20}$ and the methods used to initially derive them (ie, cadaveric dissection versus computed tomography), with this clearly demonstrated by the disparity of BSP data used in previous studies on lumbar kinetics (Tables 2 and $3)$. However, the extent to which this variation in input data affects the calculated kinetic joint reaction forces and moment outputs is less clear. Investigations of the sensitivity of lower limb kinetics during gait to BSP error have produced conflicting results. ${ }^{20}$ It has been reported that BSP error has both a minor ${ }^{21,22}$ and a significant ${ }^{23,24}$ influence on calculated lower limb joint kinetics. This is the first study to compare lumbar forces and moments calculated using varying BSP inputs and found that while lumbar force estimates are not affected by the four BSP methods compared in this investigation, lumbar moment estimations are. This makes direct comparison of reported lumbar moments inappropriate between studies that have used different BSPs data sets.

One limitation of the Pearsall et al (1996) data set is that they were generated from a limited sample of subjects. Further validation of these data in a larger sample population would be beneficial. Furthermore, as there is a substantial amount of variability in inertial characteristics of the body between individuals, ${ }^{14}$ all BSPs based on experimental sample populations involve some degree of error. No conclusive recommendation can be made as to which of these BSPs results in the most accurate lumbar kinetic data. Undoubtedly, BSP error is only one source of uncertainty in the calculation of joint kinetics, and factors that contribute to error in segment acceleration data also play an important role. ${ }^{25}$ Future studies should assess the effect of kinematic measurement error, which may occur due to marker placement error and skin movement artifact, on lumbar kinetics.

In conclusion, Pearsall's BSPs ${ }^{14}$ are the only available data that allow the estimation of clearly delineated lumbar and pelvic regions. Given that standardization is necessary to facilitate shared information and to compare lumbar loads across different populations and activities, it is recommended that future lumbo-pelvic kinetics are estimated using this BSP data set. 
Anterioposterior force

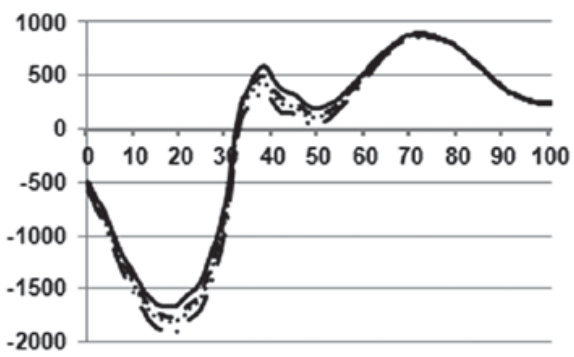

Vertical force

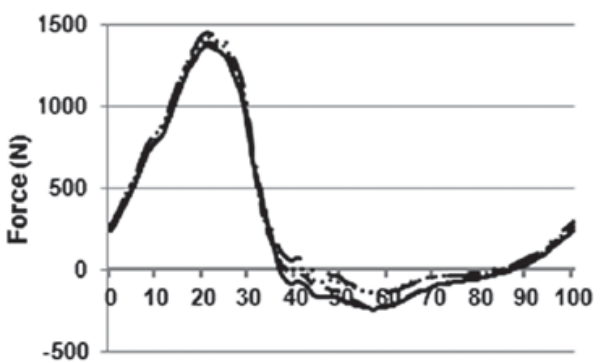

Mediolateral force

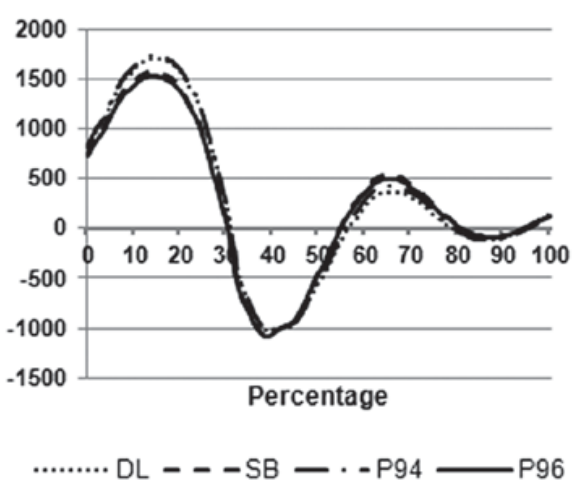

Flexion-extension moment

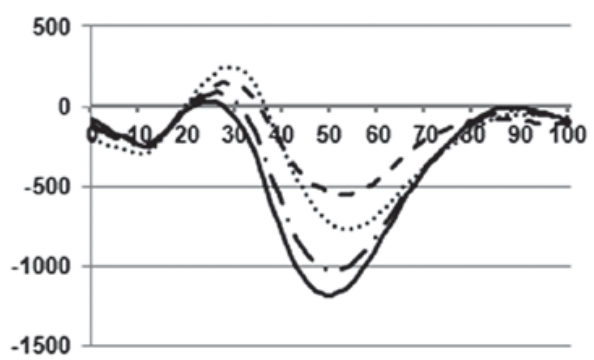

Lateral flexion moment

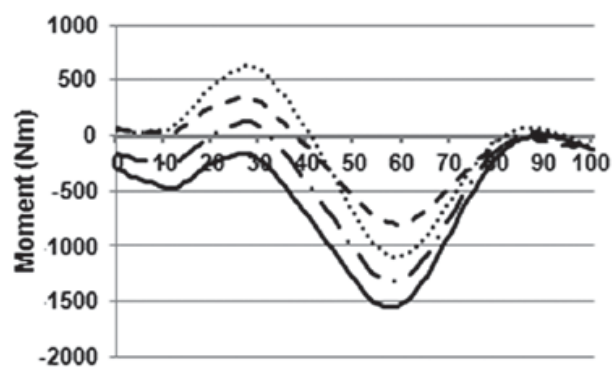

Rotation moment

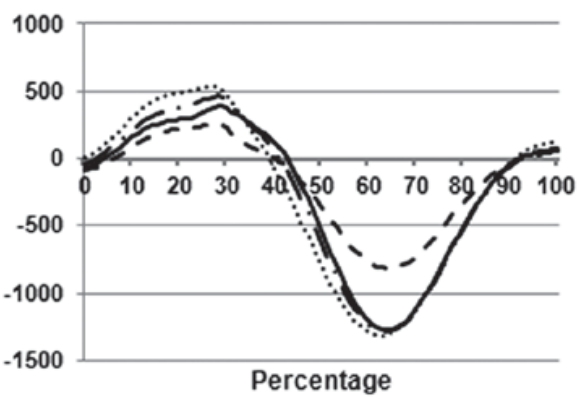

$\mathrm{DL}--\mathrm{SB}-\mathrm{-P} 94-\mathrm{P} 96$

Figure 2 - Lumbar forces and moments between front foot contact and ball release during the delivery stride, calculated using the four BSP data sets. DL, de Leva (1996); SB, Shan et al (2003); P94, Pearsall et al (1994); P96, Pearsall et al (1996).

\section{References}

1. Standaert CJ, Herring SA. Spondylolysis: a critical review. Br J Sports Med. 2000;34(6):415-422. PubMed doi:10.1136/bjsm.34.6.415

2. Brukner P, Kahn K. Clinical Sports Medicine. 3rd ed. Sydney: McGraw-Hill; 2007.

3. Foster D, John D, Elliott B, Ackland T, Fitch K. Back injuries to fast bowlers in cricket: a prospective study. $\mathrm{Br}$ J Sports Med. 1989;23(3):150-154. PubMed doi:10.1136/ bjsm.23.3.150

4. McGill SM. The biomechanics of low back injury: Implications on current practice in industry and the clinic. $J$ Biomech. 1997;30(5):465-475. PubMed doi:10.1016/ S0021-9290(96)00172-8

5. Whiting WC, Zernicke RF. Biomechanics of Musculoskeletal Injury. 2nd ed. Champaign: Human Kinetics; 2008.
6. Seay J, Selbie WS, Hamill J. In vivo lumbo-sacral forces and moments during constant speed running at different stride lengths. J Sports Sci. 2008;26(14):1519-1529. PubMed doi:10.1080/02640410802298235

7. Shum GL, Crosbie J, Lee RY. Three-dimensional kinetics of the lumbar spine and hips in low back pain patients during sit-to-stand and stand-to-sit. Spine. 2007;32(7):E211-E219. PubMed doi:10.1097/01. brs.0000259204.05598.10

8. Khoo BCC, Goh JCH, Bose K. A biomechanical model to determine lumbosacral loads during single stance phase in normal gait. Med Eng Phys. 1995;17(1):27-35. PubMed doi:10.1016/1350-4533(95)90374-K

9. Ferdinands RED, Kersting U, Marshall RN. Threedimensional lumbar segment kinetics of fast bowling in cricket. J Biomech. 2009;42(11):1616-1621. PubMed doi:10.1016/j.jbiomech.2009.04.035 
10. Dempster WT. Space requirements of the seated operator. Wright Patterson Air Force Base; 1955.

11. Zatsiorsky V, Seluyanov V, Chugunova I. In vivo body segment inertial parameters determination using a gammascanner method. Paper presented at: Study Institute and Conference of Biomechanics of Human Movement: Applications in Rehabilitation, Sports and Ergonomics. 1986; Formia, Italy.

12. O'Rahilly R, Muller F. Basic human anatomy: a regional study of human structure. Saunders; 1983.

13. Reese NB, Bandy WD, Yates C. Joint range of motion and muscle length testing. 2nd ed. St Louis: Saunders, Elsevier Inc.; 2010.

14. Pearsall DJ, Reid JG, Livingston LA. Segmental inertial parameters of the human trunk as determined from computed tomography. Ann Biomed Eng. 1996;24(2):198-210. PubMed doi:10.1007/BF02667349

15. De Leva P. Adjustments to Zatsiorky-Seluyanov's segment inertia parameters. J Biomech. 1996;29(9):1223-1230. PubMed doi:10.1016/0021-9290(95)00178-6

16. Shan G, Bohn C. Anthropometrical data and coefficients of regression related to gender and race. Appl Ergon. 2003;34(4):327-337. PubMed doi:10.1016/S00036870(03)00040-1

17. Pearsall DJ, Reid JG, Ross R. Inertial properties of the human trunk of males determined from magnetic resonance imaging. Ann Biomed Eng. 1994;22(6):692-706. PubMed doi:10.1007/BF02368294
18. Besier TF, Sturnieks DL, Alderson JA, Lloyd DG. Repeatability of gait data using a functional hip joint centre and a mean helical knee axis. J Biomech. 2003;36(8):1159-1168. PubMed doi:10.1016/S0021-9290(03)00087-3

19. Winter DA. Biomechanics and motor control of human movement. 2nd ed. New York: John Wiley \& Sons; 1990.

20. Rao G, Amarantini D, Berton E, Favier D. Influence of body segments' parameters estimation models on inverse dyamics solutions during gait. J Biomech. 2006;39(8):1531-1536. PubMed doi:10.1016/j.jbiomech.2005.04.014

21. Pearsall DJ, Costigan PA. The effect of segment parameter error on gait analysis results. Gait Posture. 1999;9(3):173183.

22. Ganley KJ, Powers CM. Determination of lower extremity anthropometric parameters using dual energy X-ray absorptiometry: the influence on net joint moments during gait. Clin Biomech (Bristol, Avon). 2004;19(2):50-56.

23. Andrews JG, Mish SP. Methods for investigating the sensitivity of joint resultants to body segment parameter variations. J. Biomech. 1996;29(5):651-654.

24. Silva MPT, Ambrosio JAC. Sensitivity of the results produced by the inverse dynamic analysis of a human stride to perturbed input data. Gait Posture. 2004;19(1):35-49.

25. Lariviere C, Gagnon D. The L5/S1 joint moment sensitivity to measurment errors in dynamic 3D multisegment lifting models. Hum Mov Sci. 1999;18(4):573-587. doi:10.1016/ S0167-9457(99)00003-2 